\title{
Erratum to: Insights into the design and interpretation of iCLIP experiments
}

\author{
Nejc Haberman ${ }^{1,2+}$, Ina Huppertz ${ }^{1,3,4+}$, Jan Attig ${ }^{1,2}$, Julian König ${ }^{1,5}$, Zhen Wang ${ }^{6,7}$, Christian Hauer ${ }^{4,8,9}$, \\ Matthias W. Hentze ${ }^{4,8}$, Andreas E. Kulozik ${ }^{8,9}$, Hervé Le Hir ${ }^{6,7}$, Tomaž Curk ${ }^{10}$, Christopher R. Sibley ${ }^{1,11}$, \\ Kathi Zarnack ${ }^{12^{*}}$ and Jernej Ule $e^{1,2^{*}}$
}

\section{Erratum}

Upon publication of the original article [1], it was noted that data submitted by the authors was accidentally omitted during typesetting. The following section of the submitted manuscript was wrongfully omitted from the Methods section in the published article [1]:

\section{Availability of data and materials}

All iCLIP data that were newly generated for this manuscript are made available at http://www.ebi.ac.uk/arrayexpress/ via accession numbers E-MTAB-5027 (PTBP1-iCLIP2), E-MTAB-5026 (PTBP1-iCLIP3), E-MTAB-3618 (eIF4A3iCLIP2) and E-MTAB-4000 (eIF4A3-iCLIP3).

This has now been acknowledged and corrected in this erratum.

The publisher apologises for these errors.
Published online: 15 August 2017

\section{Reference}

1. Haberman N, Huppertz I, Attig J, König J, Wang Z, Hauer C, Hentze MW, Kulozik AE, Le Hir H, Curk T, Sibley CR. Insights into the design and interpretation of iCLIP experiments. Genome Biol. 2017;18(1):7.

\begin{abstract}
Author details
'Department of Molecular Neuroscience, UCL Institute of Neurology, Queen Square, London WC1N 3BG, UK. ${ }^{2}$ The Crick Institute, 1 Midland Road, London NW1 1AT, UK. ${ }^{3}$ MRC Laboratory of Molecular Biology, Francis Crick Avenue, Cambridge $\mathrm{CB} 2 \mathrm{OQH}, \mathrm{UK} .{ }^{4}$ European Molecular Biology Laboratory (EMBL), Meyerhofstrasse 1, 69117 Heidelberg, Germany. ${ }^{5}$ Institute of Molecular Biology (IMB), Ackermannweg 4, 55128 Mainz, Germany. Institut de Biologie de I'ENS (IBENS), Paris, France. ${ }^{7}$ CNRS UMR 8197, Paris, Cedex 05 75230, France. ${ }^{8}$ Molecular Medicine Partnership Unit (MMPU), Im Neuenheimer Feld 350, 69120 Heidelberg, Germany. ${ }^{9}$ Department of Pediatric Oncology, Hematology and Immunology, University of Heidelberg, Im Neuenheimer Feld 430, 69120 Heidelberg, Germany. ${ }^{10}$ Faculty of Computer and Information Science, University of Ljubljana, Tržaška cesta 25, 1000 Ljubljana, Slovenia. ${ }^{11}$ Division of Brain Sciences, Department of Medicine, Imperial College London, London, UK. ${ }^{12}$ Buchmann Institute for Molecular Life Sciences (BMLS), Goethe University Frankfurt, Max-von-Laue-Str. 15, 60438 Frankfurt, Germany.
\end{abstract}

\footnotetext{
* Correspondence: kathi.zarnack@bmls.de; j.ule@ucl.ac.uk

${ }^{\dagger}$ Equal contributors

${ }^{12}$ Buchmann Institute for Molecular Life Sciences (BMLS), Goethe University Frankfurt, Max-von-Laue-Str. 15, 60438 Frankfurt, Germany

${ }^{1}$ Department of Molecular Neuroscience, UCL Institute of Neurology, Queen Square, London WC1N 3BG, UK

Full list of author information is available at the end of the article
}

(c) The Author(s). 2017 Open Access This article is distributed under the terms of the Creative Commons Attribution 4.0 International License (http://creativecommons.org/licenses/by/4.0/), which permits unrestricted use, distribution, and reproduction in any medium, provided you give appropriate credit to the original author(s) and the source, provide a link to the Creative Commons license, and indicate if changes were made. The Creative Commons Public Domain Dedication waiver (http://creativecommons.org/publicdomain/zero/1.0/) applies to the data made available in this article, unless otherwise stated. 